\title{
Deepening of response to prostate-specific membrane antigen ligand-targeted radioligand therapy beyond end of treatment
}

\author{
Thorsten Derlin $^{1}$ (D) $\cdot$ Elke Krischke $^{1} \cdot$ Tobias L. Ross $^{1} \cdot$ Frank M. Bengel $^{1}$ \\ Received: 15 February 2021 / Accepted: 23 March 2021 / Published online: 2 April 2021 \\ (C) The Author(s) 2021
}

Prostate-specific membrane antigen (PSMA)-targeted radioligand therapy (RLT) has demonstrated high anti-tumor activity in advanced-stage, metastatic castration-resistant prostate cancer (mCRPC) [1, 2].

Here, we report continued long-term anti-tumor effects after discontinuation of RLT in an 88-year-old patient with progressing disseminated bone and lymph node metastases (a) following therapy with leuprorelin acetate, abiraterone acetate, docetaxel, and denosumab. He received a total of 4 cycles of $\left[{ }^{177} \mathrm{Lu}\right] \mathrm{Lu}-\mathrm{PSMA}-617$ RLT between August 2018 and January 2019 (b). Follow-up PET/CT (c) demonstrated a good partial remission with several PSMAexpressing residual bone metastases, and the PSA was $7.6 \mu \mathrm{g} / \mathrm{L}$. He then had to discontinue RLT due to denosumab-related osteonecrosis of the jaw requiring extensive surgical treatment. Following a complicated clinical course with prolonged infection, he did not resume RLT, since PSA continued to decline without any tumor-specific treatment apart from continued leuprorelin acetate (August 2019: $0.43 \mu \mathrm{g} / \mathrm{L}$; February 2020: $0.16 \mu \mathrm{g} / \mathrm{L}$; August 2020: $0.05 \mu \mathrm{g} / \mathrm{L})$. Follow-up demonstrated complete remission in PET imaging (d) and further PSA decline to the detection limit (February 2021: $0.03 \mu \mathrm{g} / \mathrm{L}$ ).

A small subset of patients may achieve long-term complete remission after both ${ }^{225} \mathrm{Ac}$ - and ${ }^{177} \mathrm{Lu}$-labeled PSMA-617 if having undetectable PSA after the last RLT [2-4]. Usually, however, patients eventually show PSA progression [2]. The observed continuously deepening response after discontinuation of RLT provides a rationale for further research exploring the optimal duration of PSMA-targeted RLT and predictors identifying candidates for treatment de-intensification or termination. Immune mechanisms similar to using radiation therapy to augment responses to immunotherapy via the abscopal effect in mCRPC may be involved [5].

This article is part of the Topical Collection on Image of the month

Thorsten Derlin

Derlin.Thorsten@mh-hannover.de

1 Department of Nuclear Medicine, Hannover Medical School, Carl-Neuberg-Str. 1, 30625 Hannover, Germany 


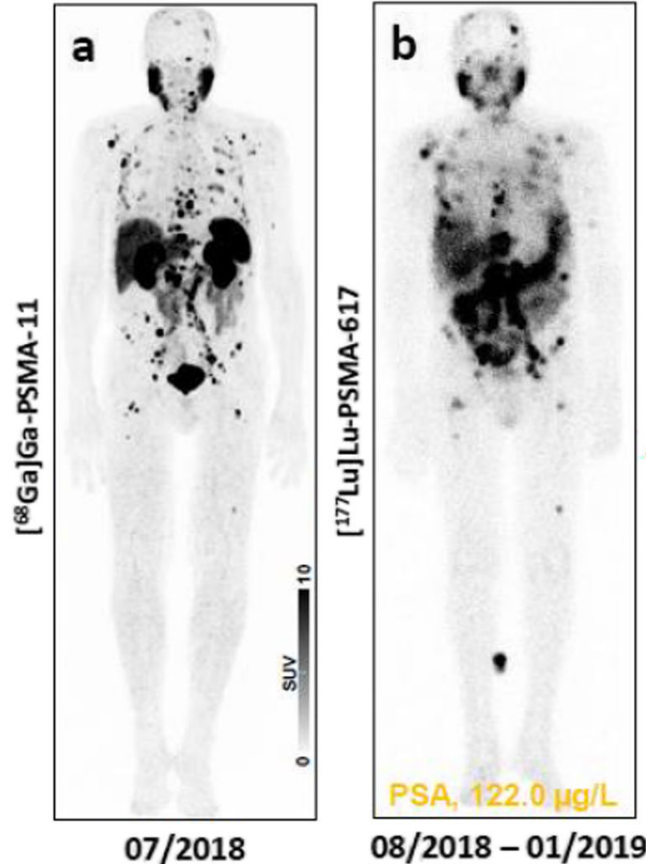

Funding Open Access funding enabled and organized by Projekt DEAL.

\section{Declarations}

Ethics approval and consent to participate All procedures performed in studies involving human participants were in accordance with the ethical standards of the institutional research committee and with the 1964 Helsinki declaration and its later amendments or comparable ethical standards. Informed consent was obtained from all individual participants included in the study.

\section{Conflict of interest The authors declare no competing interests.}

Open Access This article is licensed under a Creative Commons Attribution 4.0 International License, which permits use, sharing, adaptation, distribution and reproduction in any medium or format, as long as you give appropriate credit to the original author(s) and the source, provide a link to the Creative Commons licence, and indicate if changes were made. The images or other third party material in this article are included in the article's Creative Commons licence, unless indicated otherwise in a credit line to the material. If material is not included in the article's Creative Commons licence and your intended use is not permitted by statutory regulation or exceeds the permitted use, you will need to obtain permission directly from the copyright holder. To view a copy of this licence, visit http://creativecommons.org/licenses/by/4.0/.

\section{References}

1. Hofman MS, Violet J, Hicks RJ, et al. [177Lu]-PSMA-617 radionuclide treatment in patients with metastatic castration-resistant prostate cancer (LuPSMA trial): a single-centre, single-arm, phase 2 study. Lancet Oncol. 2018;19:825-33.

2. Violet J, Sandhu S, Iravani A, et al. Long-term follow-up and outcomes of retreatment in an expanded 50-patient single-center phase II prospective trial of 177 Lu-PSMA-617 theranostics in metastatic castration-resistant prostate cancer. J Nucl Med. 2020;61:857-65.

3. Sathekge M, Bruchertseifer F, Knoesen O, et al. ${ }^{225}$ Ac-PSMA-617 in chemotherapy-naive patients with advanced prostate cancer: a pilot study. Eur J Nucl Med Mol Imaging. 2019;46:129-38.

4. Rathke H, Bruchertseifer F, Kratochwil C, et al. First patient exceeding 5-year complete remission after ${ }^{225}$ Ac-PSMA-TAT. Eur J Nucl Med Mol Imaging. 2021;48:311-2.

5. Han HJ, Li YR, Roach M 3rd, Aggarwal R. Dramatic response to combination pembrolizumab and radiation in metastatic castration resistant prostate cancer. Ther Adv Med Oncol. 2020;12: 1758835920936084.

Publisher's note Springer Nature remains neutral with regard to jurisdictional claims in published maps and institutional affiliations. 